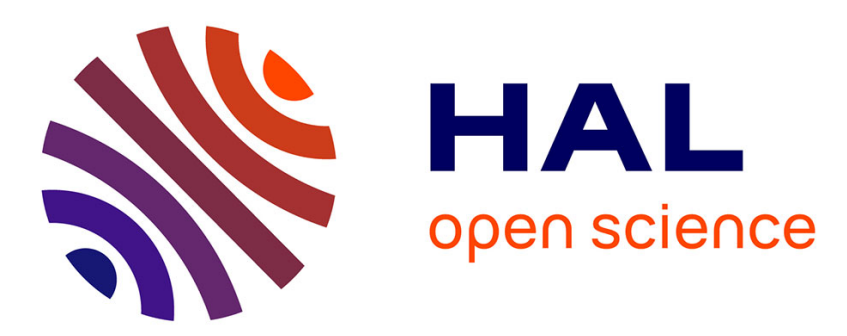

\title{
Dual Hermite subdivision schemes of de Rham-type
}

Costanza Conti, Jean-Louis Merrien, Lucia Romani

\section{To cite this version:}

Costanza Conti, Jean-Louis Merrien, Lucia Romani. Dual Hermite subdivision schemes of de Rhamtype. BIT Numerical Mathematics, 2014, 54 (4), pp.955-977. 10.1007/s10543-014-0495-z . hal01245720

\section{HAL Id: hal-01245720 \\ https://hal.science/hal-01245720}

Submitted on 18 Dec 2015

HAL is a multi-disciplinary open access archive for the deposit and dissemination of scientific research documents, whether they are published or not. The documents may come from teaching and research institutions in France or abroad, or from public or private research centers.
L'archive ouverte pluridisciplinaire HAL, est destinée au dépôt et à la diffusion de documents scientifiques de niveau recherche, publiés ou non, émanant des établissements d'enseignement et de recherche français ou étrangers, des laboratoires publics ou privés. 


\title{
Dual Hermite Subdivision Schemes of de Rham-type
}

\author{
Costanza Conti, Jean-Louis Merrien†, and Lucia Romani ${ }^{\ddagger}$
}

April 22, 2014

\begin{abstract}
Though a Hermite subdivision scheme is non-stationary by nature, its non-stationarity can be of two types, making useful the distinction between Inherently Stationary (I.S.) and Inherently Non-Stationary (I.N.S.) Hermite subdivision schemes. This paper focuses on the class of inherently stationary, dual non-interpolatory Hermite subdivision schemes that can be obtained from known Hermite interpolatory ones, by applying a generalization of the de Rham corner cutting strategy. Exploiting specific tools for the analysis of inherently stationary Hermite subdivision schemes we show that, giving up the interpolation condition, the smoothness of the associated basic limit function can be increased by one, while its support width is only enlarged by one. To accomplish the analysis of de Rham-type Hermite subdivision schemes two new theoretical results are derived and the new notion of $H C^{\ell}$-convergence is introduced. It allows the construction of Hermite-type subdivision schemes of order $d+1$ with the first element of the vector valued limit function having regularity $\ell \geq d$.
\end{abstract}

keywords:Vector subdivision, Inherently stationary Hermite subdivision, de Rham strategy, Dual parametrization, Convergence and smoothness analysis.

\section{Introduction}

Vector subdivision schemes are iterative algorithms that, starting from an initial set of vector data $f_{0}:=\left\{\mathbf{f}_{0}(\alpha), \alpha \in \mathbb{Z}\right\} \in \ell^{d+1}(\mathbb{Z})$, allow to generate denser and denser sets of vector data $\left\{f_{n+1}, n \geq 0\right\}$ through the repeated application of subdivision operators $\left\{S_{a_{n}}, n \geq 0\right\}$. The level-n subdivision operator $S_{a_{n}}$ which maps the sequence of vectors $f_{n}:=\left\{\mathbf{f}_{n}(\alpha), \alpha \in \mathbb{Z}\right\} \in \ell^{d+1}(\mathbb{Z})$ into the denser sequence of vectors $f_{n+1}:=\left\{\mathbf{f}_{n+1}(\alpha), \alpha \in \mathbb{Z}\right\} \in \ell^{d+1}(\mathbb{Z})$ is defined as

$$
\mathbf{f}_{n+1}(\alpha):=\left(S_{x_{n}} f_{n}\right)(\alpha)=\sum_{\beta \in \mathbb{Z}} \mathbf{A}_{n}(\alpha-2 \beta) \mathbf{f}_{n}(\beta), \quad \alpha \in \mathbb{Z}
$$

Therefore the refinement rules, possibly different from level to level, are averaging rules with average coefficients given by real matrices defining the so called level-n subdivision mask

$$
\mathcal{A}_{n}:=\left\{\mathbf{A}_{n}(\alpha), \alpha \in \mathbb{Z}\right\} \in \ell^{(d+1) \times(d+1)}(\mathbb{Z}) .
$$

${ }^{*}$ Costanza Conti, Dipartimento di Ingegneria Industriale, Università di Firenze, Viale Morgagni 40/44, 50134 Firenze, Italy.costanza.conti@unifi.it

${ }^{\dagger}$ Jean-Louis Merrien, INSA de Rennes, 20 avenue des Buttes de Coesmes, CS 14315, 35043 Rennes, France. jmerrien@insa-rennes.fr

${ }^{\ddagger}$ Lucia Romani, Dipartimento di Matematica e Applicazioni, Università di Milano-Bicocca, Via R. Cozzi 55, 20125 Milano, Italy. lucia.romani@unimib.it 
Whenever convergent, a vector subdivision scheme defines a continuous vector limit function for any choice of initial data $f_{0}$. In order to formally define a notion of convergence, we need to attach the data $f_{n}$, generated at the $n$-th step of the subdivision procedure, to the real parameter values $t_{n}=\left\{t_{n}(\alpha), \alpha \in \mathbb{Z}\right\}$ with $t_{n}(\alpha)<t_{n}(\alpha+1)$ and $t_{n}(\alpha+1)-t_{n}(\alpha)=2^{-n}, n \geq 0$. In this way, the vector subdivision scheme $\left\{S_{\mathbb{A}_{n}}, n \geq 0\right\}$ is said to be convergent in $\ell_{\infty}^{d+1}(\mathbb{Z})$ if for any initial vector sequence $f \in \ell_{\infty}^{d+1}(\mathbb{Z})$ there exists a continuous vector function $\mathbf{g}_{f}: \mathbb{R} \rightarrow \mathbb{R}^{d+1}$, such that for any compact $K \subset \mathbb{R}$

$$
\lim _{n \rightarrow \infty} \max _{\alpha \in \mathbb{Z} \cap 2^{n} K}\left\|\mathbf{g}_{f}\left(t_{n}(\alpha)\right)-\left(S_{\nexists_{n-1}} \cdots S_{\Re_{0}} f\right)(\alpha)\right\|_{\infty}=0
$$

and $\mathbf{g}_{f} \neq \mathbf{0}$ for some initial vector data $f$. Moreover, a vector subdivision scheme is said to be contractive if $\mathbf{g}_{f}=\mathbf{0}$ for any initial $f$, and it is called $C^{\ell}$-convergent if $\mathbf{g}_{f} \in C^{\ell}\left(\mathbb{R}, \mathbb{R}^{d+1}\right)$ for any initial vector data.

We also recall that, when the initial data is the matrix sequence

$$
\boldsymbol{\delta}_{I_{d+1}}=\left\{\delta_{I_{d+1}}(\alpha), \alpha \in \mathbb{Z}\right\} \quad \text { with } \quad \delta_{I_{d+1}}(\alpha)= \begin{cases}I_{d+1}, & \text { if } \alpha=0, \\ 0, & \text { if } \alpha \in \mathbb{Z} \backslash\{0\},\end{cases}
$$

then the limit of the vector subdivision scheme is the so called basic matrix limit function, hereinafter denoted by $\boldsymbol{\Phi}: \mathbb{R} \rightarrow \mathbb{R}^{(d+1) \times(d+1)}$.

Vector subdivision schemes, natural extension of scalar subdivision schemes, play an important role in the analysis of multivariate subdivision schemes [1] when dealing with refinable spaces generated by more than one function (see, e.g., $[2,4,7,19]$ ), as well as in the analysis of Hermite subdivision schemes. The latter are a special type of vector subdivision schemes aimed at refining vector valued sequences with the understanding that, with some extent, the $(k+1)$-th component of such a sequence corresponds to the $k$-th derivative of an underlined function (see, for instance, [11, 14, 18]). The latter fact makes a Hermite subdivision scheme non-stationary by nature: refining vector valued sequences approaching derivatives at refined grids requires to involve level dependent matrices.

Vector Hermite-type subdivision schemes are the main topic of this paper. A first contribution of our work consists in the classification of this category of schemes. In fact, though a Hermite subdivision scheme is non-stationary by nature, its non-stationarity can be of two types, making useful the distinction between Inherently Stationary (I.S.) and Inherently Non-Stationary (I.N.S.) Hermite subdivision schemes. When classifying Hermite-type subdivision schemes, the difference between interpolatory and non-interpolatory (or approximating) ones should be also considered. Starting with an initial set of Hermite-type data (i.e. vectors containing function values and associated derivatives) interpolatory Hermite subdivision schemes are able to simultaneously design points and associated derivatives, thus defining, in the limit, a continuous vector function with each component being the derivative of the previous one. Moreover, they are designed such that the initial set of vector data is interpolated as well as all sets of vector data generated by the subdivision process. On the contrary, non-interpolatory or approximating Hermite subdivision schemes, while still generating a continuous vector function with each component being an approximation of the derivative of the previous one, provide in the limit a vector function which is only approximating the initial set of vector data. Such schemes, also investigated for example in [15], turn out to be of interest whenever the interpolatory case produces "unfair" limit functions with unwanted wiggles or undulations, as well as when non-exact data are available.

In this paper we present a novel idea for the construction of non-interpolatory Hermite subdivision schemes, based on a generalization of the de Rham corner cutting strategy [8]. This is why the resulting schemes are called Hermite subdivision schemes of de Rham-type. Other examples of application of the de Rham strategy can be also found in the previous literature. In particular, in [6] 
the de Rham construction is applied in a more general but scalar context, while in [9] the Hermite case was tackled for the first time. The de Rham construction considered in this paper, at each subdivision step consists in the double application of an interpolatory Hermite subdivision operator followed by a decimation step selecting only the odd elements of the resulting sequence. Therefore, the de Rham strategy provides Hermite subdivision schemes which are dual by construction, hence approximating the initial data rather than interpolating them. We recall that, from a geometric point of view, primal vector subdivision schemes are those that at each iteration retain or modify the given vectors and create a "new" vector in between two "old" ones. Dual schemes, instead, discard all given vectors after creating two "new" ones in between any pair of them. This fact is algebraically connected with the choice of the parameter values $t_{n}:=\left\{t_{n}(\alpha), \alpha \in \mathbb{Z}\right\}$ (see, e.g, [3] for more details). More precisely, the primal parametrization is such that

$$
t_{n}(\alpha):=\frac{\alpha}{2^{n}}, \quad \alpha \in \mathbb{Z}, \quad n \geq 0
$$

On the contrary, the dual parametrization is given by

$$
t_{n}(\alpha):=\frac{\alpha-1 / 2}{2^{n}}, \quad \alpha \in \mathbb{Z}, \quad n \geq 0 .
$$

Another contribution of this paper consists in the derivation of two new theoretical results (extension of the results in [9]) for the analysis of de Rham-type Hermite subdivision schemes. Furthermore, the new notion of $\mathrm{HC}^{\ell}$-convergence is introduced to allow the construction of Hermite schemes of order $d+1$ with the first element of the vector valued limit function of regularity $\ell \geq d$.

The organization of the paper is as follows: Section 2 provides the most general formulation of vector Hermite-type subdivision schemes together with the definition of $H C^{\ell}$-convergence. Section 3 characterizes the subclass of Inherently Stationary (I.S.) Hermite subdivision schemes and recalls specific tools for the analysis of this type of Hermite schemes. Interpolatory versus dual approximating Hermite subdivision schemes are discussed in Section 4 where the so called de Rham-type construction is detailed and two new theoretical results for the analysis of de Rham-type Hermite subdivision schemes are given. The closing Section 5 illustrates applications of the de Rham-type approach: two new dual non-interpolatory subdivision schemes obtained when starting with Merrien's interpolatory schemes in $[16,17]$ are presented. For these schemes a complete smoothness analysis is given and explicative pictures are provided.

In all sections the following notation is used: vectors in $\mathbb{R}^{d+1}$ are labeled by lowercase boldface letters as $\mathbf{y}$; matrices in $\mathbb{R}^{(d+1) \times(d+1)}$ are written as uppercase boldface letters, like $\mathbf{A}$; the space of polynomials of degree at most $n$ is denoted by $\mathcal{P}_{n}$; sequences of vectors in $\mathbb{R}^{d+1}$ are denoted by lowercase calligraphic letters as $f:=\{\mathbf{f}(\alpha), \alpha \in \mathbb{Z}\}$ and the space of such sequences is denoted by $\ell^{d+1}(\mathbb{Z})$ and by $\ell_{\infty}^{d+1}(\mathbb{Z})$ if their infinity norm is bounded; sequences of matrices in $\mathbb{R}^{(d+1) \times(d+1)}$ are denoted by uppercase calligraphic letters as $\mathcal{A}:=\{\mathbf{A}(\alpha), \alpha \in \mathbb{Z}\}$, and the space of such sequences is denoted by $\ell^{(d+1) \times(d+1)}(\mathbb{Z})$. The symbol $\|\cdot\|_{\infty}$ is to denote the infinity norm of a matrix, a function or a sequence.

\section{Hermite subdivision schemes}

Hermite subdivision schemes of order $d+1$ simultaneously design function values and associated derivatives so that their limit is a continuous vector function $\mathbb{R} \rightarrow \mathbb{R}^{d+1}$ with each component being the derivative of the previous one. Since such subdivision schemes generate finer and finer sequences of "Hermite-type" data $f_{n}$ which are associated with "shifted" versions of dyadic parameters, the 
first component of $\mathbf{f}_{n}(\alpha)$ can be interpreted as getting closer to the value of a function $\psi_{n}$ at a parameter $t_{n}(\alpha)$, while the next one describes the value of its first derivative at the same location, i.e. $\psi_{n}^{(1)}\left(t_{n}(\alpha)\right)$, and so on up to the last one describing $\psi_{n}^{(d)}\left(t_{n}(\alpha)\right)$. This is why for any Hermite subdivision scheme the relation between $\mathbf{D}^{n+1} f_{n+1}$ and $\mathbf{D}^{n} f_{n}$ is given by the rule

$$
\mathbf{D}^{n+1} f_{n+1}=S_{\Re_{n}} \mathbf{D}^{n} f_{n}=\sum_{\beta \in \mathbb{Z}} \mathbf{A}_{n}(\cdot-2 \beta) \mathbf{D}^{n} \mathbf{f}_{n}(\beta), \quad n \geq 0
$$

where $\mathcal{A}_{n}:=\left\{\mathbf{A}_{n}(\alpha), \alpha \in \mathbb{Z}\right\}$ is the level- $n$ mask and $\mathbf{D}$ is the diagonal matrix with diagonal entries $2^{-j}, j=0, \ldots, d$. Obviously, (3) can also be written in a more standard way as

$$
f_{n+1}=\sum_{\beta \in \mathbb{Z}} \mathbf{D}^{-(n+1)} \mathbf{A}_{n}(\cdot-2 \beta) \mathbf{D}^{n} \mathbf{f}_{n}(\beta), \quad n \geq 0
$$

namely in terms of a level- $n$ subdivision operator $S_{\widehat{\nexists}_{n}}$ associated to the level- $n$ mask

$$
\widehat{\mathcal{A}}_{n}:=\left\{\mathbf{D}^{-(n+1)} \mathbf{A}_{n}(\alpha) \mathbf{D}^{n}, \alpha \in \mathbb{Z}\right\} .
$$

To simplify notation, from now on a Hermite subdivision scheme based on (5) will be simply denoted by $H_{\left\{\Re_{n}, n \geq 0\right\}}$.

Due to the fact that the limit vector function of any reasonable Hermite subdivision scheme must be of special type (as described at the beginning of this section), the following new and more specialized definition of $\mathrm{HC}$-convergence is given.

Definition 1 A Hermite subdivision scheme $H_{\left\{a_{n}, n \geq 0\right\}}$ acting on vectors in $\ell^{d+1}(\mathbb{Z})$ is called $H^{\ell}-$ convergent with $\ell \geq d$ if for any initial vector sequence $f_{0} \in \ell^{d+1}(\mathbb{Z})$ and the corresponding sequences of refinements $\left\{f_{n}, n \geq 0\right\}$, there exists a vector function $\phi=\left[\phi^{[i]}\right]_{i=0, \ldots, d} \in C^{\ell-d}\left(\mathbb{R}, \mathbb{R}^{d+1}\right)$ with $\phi^{[0]} \in C^{\ell}(\mathbb{R}, \mathbb{R})$ such that for any compact $K \subset \mathbb{R}$

$$
\lim _{n \rightarrow \infty} \max _{\alpha \in \mathbb{Z} \cap 2^{n} K}\left\|\mathbf{f}_{n}(\alpha)-\phi\left(2^{-n} \alpha\right)\right\|_{\infty}=0 \quad \text { and } \quad \phi^{[i]}=\frac{d^{i} \phi^{[0]}}{d x^{i}}, i=1, \ldots, d .
$$

Remark 1 To avoid confusion it is important to remark that, indeed, the notions of $C^{\ell}$ and $H C^{\ell}$ convergence are intrinsically different. The first notion refers to the minimal smoothness of the entries of the vector limit function, while the second to the maximal one. The reason why we did not select a unified definition is to be consistent with the existing literature on vector and Hermite subdivision schemes, respectively. Note also that the new notion of $H C^{\ell}$-convergence allows the construction of Hermite schemes of order $d+1$ with the first element of the vector valued limit function having regularity $\ell \geq d$.

\section{Inherently Stationary Hermite subdivision schemes}

From the previous section we know that Hermite subdivision schemes are essentially given by the repeated application of level-dependent subdivision operators $S_{\widehat{\nexists}_{n}}$ associated to the level- $n$ masks $\widehat{\mathcal{A}}_{n}:=\left\{\mathbf{D}^{-(n+1)} \mathbf{A}_{n}(\alpha) \mathbf{D}^{n}, \alpha \in \mathbb{Z}\right\}$. Now, since the presence of the matrices $\mathbf{D}^{n}$ is by nature, we can further classify a Hermite subdivision scheme by means of the following definition.

Definition 2 A Hermite subdivision scheme is named Inherently Stationary (I.S.) in case the masks $\mathcal{A}_{n}:=\left\{\mathbf{A}_{n}(\alpha), \alpha \in \mathbb{Z}\right\}, n \geq 0$, reduce to the same mask $\mathcal{A}=\mathcal{A}_{n}$ for all $n \geq 0$. It means that the 
subdivision step in (4) becomes

$$
f_{n+1}=\sum_{\beta \in \mathbb{Z}} \mathbf{D}^{-(n+1)} \mathbf{A}(\cdot-2 \beta) \mathbf{D}^{n} \mathbf{f}_{n}(\beta), \quad n \geq 0,
$$

or, equivalently, that the Hermite scheme is based on the repeated application of the subdivision operators $S_{\mathfrak{A}_{n}}, n \geq 0$ associated to the masks

$$
\widehat{\mathcal{A}}:=\left\{\mathbf{D}^{-(n+1)} \mathbf{A}(\alpha) \mathbf{D}^{n}, \alpha \in \mathbb{Z}\right\}, \quad n \geq 0 .
$$

Otherwise the Hermite subdivision scheme is named Inherently Non-Stationary (I.N.S.).

Remark 2 We remark that I.N.S. Hermite subdivision schemes occur whenever the first row of the basic matrix limit function $\mathbf{\Phi}$ belongs to some space of exponential polynomials (see, e.g., [5]). An example of such kind of I.N.S. Hermite subdivision schemes is presented in [20].

In accordance with the notation of the previous section, for shortness, from now on an I.S. Hermite subdivision scheme will be denoted by $H_{\mathfrak{A}}$.

In the rest of the paper we focus our attention on the class of inherently stationary Hermite subdivision schemes only. We emphasize that if an I.S. Hermite subdivision scheme $H_{\Re}$ defining the vector sequences $\left\{f_{n}, n \geq 0\right\}$ converges in the sense specified in Definition 1 , then the sequence $\mathbf{D}^{n} f_{n}$ converges to the vector function $\left[\phi^{[0]}, 0 \cdots, 0\right]^{T}$. This implies the existence of a convergent stationary counterpart of any inherently stationary Hermite subdivision scheme, hereinafter denoted by $S_{\sharp}$. This is a crucial fact that we are going to use in the following for the analysis of I.S. Hermite subdivision schemes.

\subsection{Analysis tools for Inherently Stationary Hermite subdivision schemes}

We start this section with some definitions needed to the development of the forthcoming theoretical results.

Definition 3 A primal Hermite subdivision scheme $H_{\mathfrak{A}}$ is called interpolatory if, starting from any vector sequence $f_{0} \in \ell^{d+1}(\mathbb{Z})$, then $f_{n+1}(2 \cdot)=f_{n}(\cdot)$ for all $n \geq 0$. This is equivalent to the following property either of the mask $\mathcal{A}$ or of the mask $\widehat{\mathcal{A}}:=\left\{\mathbf{D}^{-(n+1)} \mathbf{A}(\alpha) \mathbf{D}^{n}, \alpha \in \mathbb{Z}\right\}$ :

$$
\mathbf{A}(0)=\mathbf{D}, \quad \widehat{\mathbf{A}}(0)=I, \quad \mathbf{A}(2 \beta)=\widehat{\mathbf{A}}(2 \beta)=\mathbf{0}, \quad \forall \beta \neq 0 .
$$

Definition 4 We say that a Hermite subdivision scheme $H_{\Re}$ reproduces a function $\varphi \in C^{d}(\mathbb{R})$ if for any initial vector sequence $f_{0}=\left\{\mathbf{f}_{0}(\alpha), \alpha \in \mathbb{Z}\right\}$ such that $\mathbf{f}_{0}(\alpha)=\left[\varphi(\alpha), \ldots, \varphi^{(d)}(\alpha)\right]^{T}$, we have $f_{n}=\left\{\mathbf{f}_{n}(\alpha), \alpha \in \mathbb{Z}\right\}$ with $\mathbf{f}_{n}(\alpha)=\left[\varphi\left(\alpha / 2^{n}\right), \ldots, \varphi^{(d)}\left(\alpha / 2^{n}\right)\right]^{T}$ for any $n \in \mathbb{N}, \alpha \in \mathbb{Z}$.

We continue by associating to any function $f \in C^{d}(\mathbb{R})$ the vector sequence $v_{f}=\left\{\mathbf{v}_{f}(\alpha), \alpha \in \mathbb{Z}\right\} \in$ $\ell^{d+1}(\mathbb{Z})$ with

$$
\mathbf{v}_{f}(\alpha):=\left[f(\alpha), f^{\prime}(\alpha), \ldots, f^{(d)}(\alpha)\right]^{T}, \quad \alpha \in \mathbb{Z} .
$$

A fundamental property of Hermite subdivision schemes is the spectral condition introduced in [10], which requires the existence of particular polynomial eigenvalues of the subdivision operator $S_{\mathfrak{A}}$, stationary counterpart of $H_{\Re}$. 
Definition 5 A stationary mask $\mathcal{A}$ or its associated subdivision operator $S_{\sharp}$ satisfies the spectral condition of order $\ell+1$ if there exist polynomials $p_{j} \in \mathcal{P}_{j}, \operatorname{deg} p_{j}=j, j=0, \ldots, \ell$, such that

$$
S_{\Omega} v_{p_{j}}=\frac{1}{2^{j}} v_{p_{j}},
$$

where $v_{p_{j}}=\left\{\mathbf{v}_{p_{j}}(\alpha), \alpha \in \mathbb{Z}\right\}, \mathbf{v}_{p_{j}}(\alpha)$ is defined as in (7) and $p_{j}$ is normalized such that $p_{j}(x)=$ $\frac{1}{j !} x^{j}+\cdots$.

Remark 3 In [10] it was proven that the spectral condition is also equivalent to the sum rule introduced by Han et al. in [13, 15].

In the following proposition we discuss a property fulfilled by the stationary counterpart of a Hermite subdivision scheme reproducing polynomials of a certain degree.

Proposition 1 Let $H_{\neg}$ be a Hermite subdivision scheme of order $d+1$, reproducing a basis of $\mathcal{P}_{\ell}$ where $\ell \geq d$. Then its stationary counterpart $S_{\Omega}$ satisfies the spectral condition of order $\ell+1$ for the sequence of polynomials $p_{j}(x)=\frac{x^{j}}{j !}, j=0, \ldots, \ell$.

Proof: If a basis of $\mathcal{P}_{\ell}$ is reproduced by $H_{\Omega}$ then, using linearity, any polynomial of $\mathcal{P}_{\ell}$ is reproduced. For $j=0, \ldots, \ell$, let $f_{0}:=v_{p_{j}}$. Using (3) at step $n=0, \mathbf{D f}_{1}(\alpha)=\sum_{\beta \in \mathbb{Z}} \mathbf{A}(\alpha-2 \beta) \mathbf{f}_{0}(\beta), \alpha \in \mathbb{Z}$. Since $p_{j}$ is reproduced by the Hermite scheme, we deduce that starting from $f_{0}=\left\{\mathbf{f}_{0}(\alpha), \alpha \in \mathbb{Z}\right\}$ with $\mathbf{f}_{0}(\alpha)=\left[p_{j}(\alpha), \ldots, p_{j}^{(d)}(\alpha)\right]^{T}$

- in case $j \leq d$,

$$
\mathbf{f}_{1}(\alpha)=\left[\begin{array}{c}
p_{j}(\alpha / 2) \\
p_{j}^{\prime}(\alpha / 2) \\
\vdots \\
p_{j}^{(j)}(\alpha / 2) \\
p_{j}^{(j+1)}(\alpha / 2) \\
\vdots \\
p_{j}^{(d)}(\alpha / 2)
\end{array}\right]=\left[\begin{array}{c}
\frac{1}{2^{j}} \frac{\alpha^{j}}{j !} \\
\frac{1}{2^{j-1}} \frac{\alpha^{j-1}}{(j-1) !} \\
\vdots \\
1 \\
0 \\
\vdots \\
0
\end{array}\right] \quad \text { and } \quad \operatorname{Df}_{1}(\alpha)=\frac{1}{2^{j}}\left[\begin{array}{c}
\frac{\alpha^{j}}{j !} \\
\frac{\alpha^{j-1}}{(j-1) !} \\
\vdots \\
1 \\
0 \\
\vdots \\
0
\end{array}\right]=\frac{1}{2^{j}} \mathbf{v}_{p_{j}}(\alpha)
$$

- while in case $j>d$,

$$
\mathbf{f}_{1}(\alpha)=\left[\begin{array}{c}
p_{j}(\alpha / 2) \\
p_{j}^{\prime}(\alpha / 2) \\
\vdots \\
p_{j}^{(d)}(\alpha / 2)
\end{array}\right]=\left[\begin{array}{c}
\frac{1}{2^{j}} \frac{\alpha^{j}}{j^{j}} \\
\frac{1}{2^{j-1}} \frac{\alpha^{j-1}}{(j-1) !} \\
\vdots \\
\frac{1}{2^{j-d}} \frac{\alpha^{j-d}}{(j-d) !}
\end{array}\right] \quad \text { and } \quad \mathbf{D f}_{1}(\alpha)=\frac{1}{2^{j}}\left[\begin{array}{c}
\frac{\alpha^{j}}{j^{j}} \\
\frac{\alpha^{j-1}}{(j-1) !} \\
\vdots \\
\frac{\alpha^{j-d}}{(j-d) !}
\end{array}\right]=\frac{1}{2^{j}} \mathbf{v}_{p_{j}}(\alpha)
$$

which is exactly the spectral condition at step $j$, i.e. $\frac{1}{2^{j}} v_{p_{j}}=\mathbf{D} f_{1}=S_{a} f_{0}=S_{a} v_{p_{j}}$.

Remark 4 In [11] it is proven that, for interpolatory Hermite subdivision schemes of order $d+1$, the reproduction of polynomials of degree at least $d$ is a necessary condition to obtain $H C^{d}$-convergence.

The next theorem recalls a factorization result proven in [18] for Hermite subdivision schemes whose stationary counterpart satisfies the spectral condition. 
Theorem 2 [18, Theorem 2] Let $H_{\Re}$ be a Hermite subdivision scheme of order $d+1$. If the mask $\mathcal{A} \in \ell^{(d+1) \times(d+1)}(\mathbb{Z})$ of its stationary counterpart $S_{\mathfrak{A}}$ satisfies the spectral condition of order at least $d+1$, then there exists a finitely supported mask $\mathcal{B}$ in $\ell^{(d+1) \times(d+1)}(\mathbb{Z})$ such that

$$
T_{d} S_{\mathfrak{A}}=2^{-d} S_{B} T_{d}
$$

where

$$
T_{d}:=\left[\begin{array}{ccccc}
\delta & -1 & \ldots & -\frac{1}{(d-1) !} & -\frac{1}{d !} \\
& \delta & \ddots & \vdots & -\frac{1}{(d-1) !} \\
& & \ddots & -1 & \vdots \\
& & & \delta & -1 \\
& & & & 1
\end{array}\right], \quad(\delta y)(\alpha)=y(\alpha+1)-y(\alpha)
$$

is the Taylor operator of order $d+1$ acting on $\ell^{d+1}(\mathbb{Z})$ and $S_{\mathfrak{B}}$ is the stationary subdivision scheme with mask $\mathcal{B}$, usually termed the Taylor subdivision scheme associated to $S_{\mathfrak{a}}$.

Following the reasoning in [18] we can now prove the following theorem that allows us to check the convergence of a Hermite subdivision scheme by studying the behavior of the associated Taylor subdivision scheme.

Theorem 3 Let $H_{\mathfrak{A}}$ be a Hermite subdivision scheme of order $d+1$. Suppose that the mask $\mathcal{A} \in$ $\ell^{(d+1) \times(d+1)}(\mathbb{Z})$ of its stationary counterpart $S_{\Re}$ satisfies the spectral condition of order at least $d+1$ and the associated Taylor subdivision scheme $S_{\mathfrak{B}}$ is $C^{k}$-convergent. Then the Hermite subdivision scheme $H_{\Re}$ is $H C^{d+k}$-convergent.

Proof: The proof follows the lines of the proof of [18, Theorem 21] and following results. We begin by using the contractivity of $S_{\mathfrak{B}}$ and properties of vector subdivision schemes to prove that there exists a continuous function $\psi \in C^{k}(\mathbb{R}, \mathbb{R})$ such that the last component of $\mathbf{f}_{n}(\alpha)=\left[f_{n}(\alpha), \cdots, f_{n}^{(d)}(\alpha)\right]^{T}$ satisfies

$$
\lim _{n \rightarrow \infty} \max _{\alpha \in \mathbb{Z} \cap 2^{n} K}\left|f_{n}^{(d)}(\alpha)-\psi\left(2^{-n} \alpha\right)\right|=0,
$$

for any compact subset $K \subset \mathbb{R}$. Then, step by step, using successive integrations (see [18, Lemma $22]$ ), we prove that each component converges. The integration gives that, at each step, we get a primitive function of the previous one so that for the first component $\varphi$ of the vector valued limit function we have $\varphi \in C^{d+k}(\mathbb{R}, \mathbb{R})$ and $\varphi^{(d)}=\psi \in C^{k}(\mathbb{R}, \mathbb{R})$.

\section{Interpolatory versus dual approximating Hermite schemes: the de Rham approach}

For an interpolatory Hermite subdivision scheme $H_{\Re}$ of order $d+1$, with mask $\widehat{\mathcal{A}}=\left\{\mathbf{D}^{-(n+1)} \mathbf{A}(\alpha) \mathbf{D}^{n}\right.$, $\alpha \in \mathbb{Z}\}$, let us define a new dual non-interpolatory Hermite subdivision scheme by taking the de Rham transform of $H_{\mathfrak{A}}$ and itself as follows (see also [9]). Starting with the data $f_{0} \in \ell^{d+1}(\mathbb{Z})$, for $n \geq 0$ we define the sequence $f_{n+1}=\left\{\mathbf{f}_{n+1}(\alpha), \alpha \in \mathbb{Z}\right\}$ by computing

$$
\begin{array}{ll}
\mathbf{D}^{n+1} \widetilde{\mathbf{f}}_{n+1}(\beta) & :=\sum_{\gamma \in \mathbb{Z}} \mathbf{A}(\beta-2 \gamma) \mathbf{D}^{n} \mathbf{f}_{n}(\gamma), \quad \beta \in \mathbb{Z} \\
\mathbf{D}^{n+2} \widetilde{\mathbf{f}}_{n+2}(\alpha) & :=\sum_{\beta \in \mathbb{Z}} \mathbf{A}(\alpha-2 \beta) \mathbf{D}^{n+1} \widetilde{\mathbf{f}}_{n+1}(\beta), \quad \alpha \in \mathbb{Z} \\
\mathbf{f}_{n+1}(\alpha) & :=\widetilde{\mathbf{f}}_{n+2}(2 \alpha+1), \quad \alpha \in \mathbb{Z} .
\end{array}
$$


Therefore

$$
\begin{aligned}
\mathbf{D}^{n+1} \mathbf{f}_{n+1}(\alpha) & =\mathbf{D}^{-1} \mathbf{D}^{n+2} \widetilde{\mathbf{f}}_{n+2}(2 \alpha+1) \\
& =\mathbf{D}^{-1} \sum_{\beta \in \mathbb{Z}} \mathbf{A}(2 \alpha+1-2 \beta) \sum_{\gamma \in \mathbb{Z}} \mathbf{A}(\beta-2 \gamma) \mathbf{D}^{n} \mathbf{f}_{n}(\gamma) \\
& =\mathbf{D}^{-1} \sum_{\gamma \in \mathbb{Z}}\left(\sum_{\beta \in \mathbb{Z}} \mathbf{A}(2 \alpha+1-2 \beta) \mathbf{A}(\beta-2 \gamma)\right) \mathbf{D}^{n} \mathbf{f}_{n}(\gamma) \\
& =\mathbf{D}^{-1} \sum_{\gamma \in \mathbb{Z}}\left(\sum_{\beta \in \mathbb{Z}} \mathbf{A}(2 \alpha+1-4 \gamma-2 \beta) \mathbf{A}(\beta)\right) \mathbf{D}^{n} \mathbf{f}_{n}(\gamma) \\
& =\sum_{\gamma \in \mathbb{Z}} \overline{\mathbf{A}}(\alpha-2 \gamma) \mathbf{D}^{n} \mathbf{f}_{n}(\gamma),
\end{aligned}
$$

where

$$
\overline{\mathbf{A}}(\alpha)=\mathbf{D}^{-1} \sum_{\beta \in \mathbb{Z}} \mathbf{A}(2 \alpha+1-2 \beta) \mathbf{A}(\beta), \quad \alpha \in \mathbb{Z} .
$$

Definition 6 For a Hermite subdivision scheme $H_{\Re}$, the associated de Rham-type Hermite subdivision scheme $H_{\overline{\mathbb{R}}}$ is the Hermite subdivision scheme having mask $\widehat{\overline{\mathcal{A}}}=\left\{\mathbf{D}^{-(n+1)} \overline{\mathbf{A}}(\alpha) \mathbf{D}^{n}, \alpha \in \mathbb{Z}\right\}$ where $\overline{\mathcal{A}}=\{\overline{\mathbf{A}}(\alpha), \alpha \in \mathbb{Z}\}$ is defined by (8).

Remark 5 We recall from [9] that, if the support of $S_{\Re}$ is $\left[\sigma, \sigma^{\prime}\right]$, then the support of its de Rham transform $S_{\bar{A}}$ is contained in $\left[(3 \sigma-1) / 2,\left(3 \sigma^{\prime}-1\right) / 2\right]$.

We continue by discussing the parametrization of the generated de Rham-type Hermite subdivision scheme. At each step, given a sequence $f_{n}$ associated to the parameter values $t_{n}$, the primal interpolatory Hermite subdivision scheme is applied twice to get the sequence associated to the parameter values

$$
\tilde{t}_{n+2}(4 \alpha):=t_{n}(\alpha), \quad \widetilde{t}_{n+2}(4 \alpha+1):=\frac{3 t_{n}(\alpha)+t_{n}(\alpha+1)}{4},
$$

and

$$
\widetilde{t}_{n+2}(4 \alpha+2):=\frac{t_{n}(\alpha)+t_{n}(\alpha+1)}{2}, \quad \widetilde{t}_{n+2}(4 \alpha+3):=\frac{t_{n}(\alpha)+3 t_{n}(\alpha+1)}{4} .
$$

The sequence $\widetilde{f}_{n+2}$ is then decimated (by taking the odd entries only) to get $f_{n+1}$, which is therefore associated to the dual parameter values

$$
t_{n+1}(2 \alpha)=\frac{3 t_{n}(\alpha)+t_{n}(\alpha+1)}{4}, \quad t_{n+1}(2 \alpha+1)=\frac{t_{n}(\alpha)+3 t_{n}(\alpha+1)}{4},
$$

meaning that the corresponding subdivision scheme is dual in the sense of (2).

We conclude this section by proving that, if the stationary counterpart of the interpolatory Hermite subdivision scheme satisfies a spectral condition of order $\ell+1$, so does the stationary counterpart of the de Rham-type Hermite subdivision scheme. This result extends [9, Theorem 5].

Theorem 4 Suppose that $S_{\mathfrak{A}}$ satisfies the spectral condition of order $\ell+1$. Then the corresponding de Rham transform $S_{\overline{\mathfrak{A}}}$, with mask $\overline{\mathcal{A}}=\{\overline{\mathbf{A}}(\alpha), \alpha \in \mathbb{Z}\}$ defined by (8), also satisfies the spectral condition of order $\ell+1$. 
Proof: $\quad$ Since $S_{\Re}$ satisfies the spectral condition of order $\ell+1$, for $i=0, \ldots, \ell$ for $p_{i} \in \mathcal{P}_{i}$, $p_{i}(x)=\frac{1}{i !} x^{i}+\cdots$ and for the sequence $v_{i}$ with elements $\mathbf{v}_{i}(\alpha)=\left[p_{i}(\alpha), \ldots, p_{i}^{(d)}(\alpha)\right]^{T}, \alpha \in \mathbb{Z}$ we know that

$$
S_{\Re} v_{i}=\frac{1}{2^{i}} v_{i} \quad \Leftrightarrow \quad \sum_{\beta \in \mathbb{Z}} \mathbf{A}(\alpha-2 \beta) \mathbf{v}_{i}(\beta)=\frac{1}{2^{i}} \mathbf{v}_{i}(\alpha), \quad \alpha \in \mathbb{Z},
$$

where, for the sake of simplicity, we omit $p_{i}$ from the lower index of the sequence $v_{i}$ (differently from Definition 5). Our aim is to show by induction that for $i=0, \ldots, \ell$ we are able to build the polynomials $\bar{p}_{i}$ with leading coefficient $1 / i$ ! and the corresponding vector sequences $\bar{v}_{i}$ such that

$$
S_{\overline{\bar{q}}} \bar{v}_{i}=\frac{1}{2^{i}} \bar{v}_{i} \quad \Leftrightarrow \quad \sum_{\beta \in \mathbb{Z}} \overline{\mathbf{A}}(\alpha-2 \beta) \overline{\mathbf{v}}_{i}(\beta)=\frac{1}{2^{i}} \overline{\mathbf{v}}_{i}(\alpha), \quad \alpha \in \mathbb{Z} .
$$

We begin by proving that (9) implies

$$
\sum_{\beta \in \mathbb{Z}} \overline{\mathbf{A}}(\alpha-2 \beta) \mathbf{v}_{j}(\beta)=\frac{1}{4^{j}} \mathbf{D}^{-1} \mathbf{v}_{j}(2 \alpha+1), \quad j=0, \ldots, \ell, \quad \alpha \in \mathbb{Z} .
$$

Indeed,

$$
\begin{aligned}
\sum_{\beta \in \mathbb{Z}} \overline{\mathbf{A}}(\alpha-2 \beta) \mathbf{v}_{j}(\beta) & =\mathbf{D}^{-1} \sum_{\beta \in \mathbb{Z}} \sum_{\gamma \in \mathbb{Z}} \mathbf{A}(2 \alpha-4 \beta-2 \gamma+1) \mathbf{A}(\gamma) \mathbf{v}_{j}(\beta) \\
& =\mathbf{D}^{-1} \sum_{\beta \in \mathbb{Z}} \sum_{\gamma^{\prime} \in \mathbb{Z}} \mathbf{A}\left(2 \alpha+1-2 \gamma^{\prime}\right) \mathbf{A}\left(\gamma^{\prime}-2 \beta\right) \mathbf{v}_{j}(\beta) \\
& =\mathbf{D}^{-1} \sum_{\gamma^{\prime} \in \mathbb{Z}} \sum_{\beta \in \mathbb{Z}} \mathbf{A}\left(2 \alpha+1-2 \gamma^{\prime}\right) \mathbf{A}\left(\gamma^{\prime}-2 \beta\right) \mathbf{v}_{j}(\beta) \\
& =\mathbf{D}^{-1} \sum_{\gamma^{\prime} \in \mathbb{Z}} \mathbf{A}\left(2 \alpha+1-2 \gamma^{\prime}\right) \frac{1}{2^{j}} \mathbf{v}_{j}\left(\gamma^{\prime}\right)=\frac{1}{4^{j}} \mathbf{D}^{-1} \mathbf{v}_{j}(2 \alpha+1)
\end{aligned}
$$

We continue proving the claim by induction on $j$ : for $j=0$, we define $\bar{p}_{0}(x)=1=p_{0}(x)$ so that $\overline{\mathbf{v}}_{0}(\alpha)=\mathbf{v}_{0}(\alpha)=[1,0, \ldots, 0]^{T}$ and from $(11)$ we get

$$
\sum_{\beta \in \mathbb{Z}} \overline{\mathbf{A}}(\alpha-2 \beta) \overline{\mathbf{v}}_{0}(\beta)=\sum_{\beta \in \mathbb{Z}} \overline{\mathbf{A}}(\alpha-2 \beta) \mathbf{v}_{0}(\beta)=\mathbf{D}^{-1} \mathbf{v}_{0}(2 \alpha+1)=\mathbf{v}_{0}(\alpha)=\overline{\mathbf{v}}_{0}(\alpha),
$$

which is $S_{\bar{\Omega}} \bar{v}_{0}=\frac{1}{2^{0}} \bar{v}_{0}$. Next, we assume that (10) is true for $i=0,1, \ldots, j-1$ where $0<j \leq \ell$. Since $\left\{p_{j}, \bar{p}_{j-1}, \ldots, \bar{p}_{0}\right\}$ is a basis of $\mathcal{P}_{j}$, and since we know that the leading coefficient of $p_{j}$ is $1 / j$ !, we can write

$$
p_{j}(2 x+1)=2^{j} p_{j}(x)+\sum_{i=0}^{j-1} \lambda_{j, i} \bar{p}_{i}(x) .
$$

Differentiating up to $d$ times both sides of the previous equation with respect to $x$ and evaluating at $\alpha$ we get

$$
\mathbf{D}^{-1} \mathbf{v}_{j}(2 \alpha+1)=2^{j} \mathbf{v}_{j}(\alpha)+\sum_{i=0}^{j-1} \lambda_{j, i} \overline{\mathbf{v}}_{i}(\alpha), \quad \alpha \in \mathbb{Z}
$$

Then, let us define $\bar{p}_{j}(x)=p_{j}(x)+\sum_{i=0}^{j-1} \mu_{j, i} \bar{p}_{j}(x)$ where $\mu_{j, i}=-\lambda_{j, i} \frac{2^{i-j}}{2^{j}-2^{i}}$ and construct the corresponding vector sequence $\bar{v}_{\bar{p}_{j}}$ that we are going to simply denote as $\bar{v}_{j}$. Note that $\bar{p}_{j}$ is a 
polynomial of degree $j$ and its leading coefficient is exactly $1 / j$ !. Therefore, differentiating $\bar{p}_{j}$ up to $d$ times and evaluating at $\alpha$, we obtain

$$
\overline{\mathbf{v}}_{j}(\alpha)=\mathbf{v}_{j}(\alpha)+\sum_{i=0}^{j-1} \mu_{j, i} \overline{\mathbf{v}}_{i}(\alpha) .
$$

Next, using the induction assumption, we observe that for $i=0,1, \ldots, j-1$,

$$
\sum_{\beta \in \mathbb{Z}} \overline{\mathbf{A}}(\alpha-2 \beta) \overline{\mathbf{v}}_{i}(\beta)=\frac{1}{2^{i}} \overline{\mathbf{v}}_{i}(\alpha), \quad \alpha \in \mathbb{Z} .
$$

Thus, due to (11), (13) and the induction hypothesis we have that

$$
\begin{aligned}
\sum_{\beta \in \mathbb{Z}} \overline{\mathbf{A}}(\alpha-2 \beta) \overline{\mathbf{v}}_{j}(\beta) & =\sum_{\beta \in \mathbb{Z}} \overline{\mathbf{A}}(\alpha-2 \beta)\left(\mathbf{v}_{j}(\beta)+\sum_{i=0}^{j-1} \mu_{j, i} \overline{\mathbf{v}}_{i}(\beta)\right) \\
& =\frac{1}{4^{j}} \mathbf{D}^{-1} \mathbf{v}_{j}(2 \alpha+1)+\sum_{i=0}^{j-1} \frac{\mu_{j, i}}{2^{i}} \overline{\mathbf{v}}_{i}(\alpha) \\
& =\frac{1}{2^{j}} \mathbf{v}_{j}(\alpha)+\sum_{i=0}^{j-1}\left(\frac{\lambda_{j, i}}{4^{j}}+\frac{\mu_{j, i}}{2^{i}}\right) \overline{\mathbf{v}}_{i}(\alpha) \quad(\text { from }(12)) \\
& =\frac{1}{2^{j}} \mathbf{v}_{j}(\alpha)+\sum_{i=0}^{j-1} \mu_{j, i}\left(-\frac{1}{4^{j}} \frac{2^{j}-2^{i}}{2^{i-j}}+\frac{1}{2^{i}}\right) \overline{\mathbf{v}}_{i}(\alpha) \\
& =\frac{1}{2^{j}}\left(\mathbf{v}_{j}(\alpha)+\sum_{i=0}^{j-1} \mu_{j, i} \overline{\mathbf{v}}_{i}(\alpha)\right)=\frac{1}{2^{j}} \overline{\mathbf{v}}_{j}(\alpha) \quad(\text { from }(13)) .
\end{aligned}
$$

We conclude that $\sum_{\beta \in \mathbb{Z}} \overline{\mathbf{A}}(\alpha-2 \beta) \overline{\mathbf{v}}_{j}(\beta)=\frac{1}{2^{j}} \overline{\mathbf{v}}_{j}(\alpha), \alpha \in \mathbb{Z}$ which is the end of induction step $j$.

As a consequence of Theorem 4, we get the following Corollary, extension of [9, Corollary 6].

Corollary 5 Suppose that $S_{\mathfrak{A}}$ satisfies the spectral condition of order $\ell+1$ with the sequence of polynomials $p_{j}(x)=x^{j} / j$ ! and corresponding vector sequences $v_{j}$ for $j=0, \ldots, \ell$. Then $S_{\bar{\lambda}}$ with mask $\overline{\mathcal{A}}=\{\overline{\mathbf{A}}(\alpha), \alpha \in \mathbb{Z}\}$ defined by (8) satisfies the spectral condition of order $\ell+1$ with the sequence of polynomials $\bar{p}_{j}(x)=(x-1 / 2)^{j} / j$ ! and corresponding vector sequences $\bar{v}_{j}$ for $j=0, \ldots, \ell$.

Proof: We use the notation of the previous theorem and prove the claim by induction.

For $j=0, p_{0}(x)=1$, and we have seen in the proof of the previous theorem that $\bar{p}_{0}(x)=1=$ $p_{0}(x-1 / 2)$. Next, we suppose that for $i=0, \ldots, j-1, \bar{p}_{i}(x)=(x-1 / 2)^{i} / i !$, and we write $p_{j}(2 x+1)$ as

$$
\begin{aligned}
p_{j}(2 x+1) & =2^{j} x^{j} / j !+2^{j}(x-1 / 2+1)^{j} / j !-2^{j}(x-1 / 2+1 / 2)^{j} / j ! \\
& =2^{j} x^{j} / j !+\frac{2^{j}}{j !} \sum_{i=0}^{j-1}\left(\begin{array}{l}
j \\
i
\end{array}\right)\left(1-1 / 2^{j-i}\right)(x-1 / 2)^{i}=2^{j} p_{j}(x)+\sum_{i=0}^{j-1} \lambda_{j, i} \bar{p}_{i}(x),
\end{aligned}
$$

with $\lambda_{j, i}=\frac{2^{j}-2^{i}}{(j-i) !}$. 
In the proof of the previous theorem we have seen that $\bar{p}_{j}(x)=p_{j}(x)+\sum_{i=0}^{j-1} \mu_{j, i} \bar{p}_{i}(x)$ where $\mu_{j, i}=-\lambda_{j, i} \frac{2^{i-j}}{2^{j}-2^{i}}$, so that we obtain

$$
\begin{aligned}
\bar{p}_{j}(x) & =p_{j}(x)-\sum_{i=0}^{j-1} \lambda_{j, i} \frac{2^{i-j}}{2^{j}-2^{i}} \bar{p}_{i}(x)=p_{j}(x)-\sum_{i=0}^{j-1} \frac{2^{j}-2^{i}}{(j-i) !} \frac{2^{i-j}}{2^{j}-2^{i}} \bar{p}_{i}(x) \\
& =\frac{\left(x-\frac{1}{2}+\frac{1}{2}\right)^{j}}{j !}-\sum_{i=0}^{j-1} \frac{2^{i-j}}{(j-i) !} \bar{p}_{i}(x) \\
& =\frac{1}{j !} \sum_{i=0}^{j}\left(\begin{array}{l}
j \\
i
\end{array}\right) 2^{i-j}(x-1 / 2)^{i}-\frac{1}{j !} \sum_{i=0}^{j-1}\left(\begin{array}{l}
j \\
i
\end{array}\right)(x-1 / 2)^{i} 2^{i-j} \\
& =(x-1 / 2)^{j} / j !
\end{aligned}
$$

and the result is proved by this finite induction.

\section{Applications}

In this section we apply the de Rham strategy to two known Hermite interpolatory subdivision schemes and obtain two new dual non-interpolatory Hermite subdivision schemes whose convergence and smoothness is investigated with the support of the theoretical results in the previous sections and of the sufficient conditions for convergence of stationary vector subdivision schemes in [1]. As a matter of fact, giving up the interpolation condition, we manage to increase by one the regularity of the basic limit function, while its support width is only enlarged by one.

\subsection{A dual non-interpolatory $H C^{2}$ Hermite subdivision scheme of order 2}

We start with the interpolatory Hermite subdivision scheme of order $d+1=2$ proposed by Merrien in [16], which depends on two free parameters $\lambda, \mu \in \mathbb{R}$. The non-zero matrices of the mask $\mathcal{A}$ of its stationary counterpart are

$$
\mathbf{A}(-1)=\left[\begin{array}{cc}
\frac{1}{2} & \lambda \\
\frac{1}{2}(1-\mu) & \frac{\mu}{4}
\end{array}\right], \quad \mathbf{A}(0)=\left[\begin{array}{ll}
1 & 0 \\
0 & \frac{1}{2}
\end{array}\right], \quad \mathbf{A}(1)=\left[\begin{array}{cc}
\frac{1}{2} & -\lambda \\
\frac{1}{2}(\mu-1) & \frac{\mu}{4}
\end{array}\right] .
$$

For any values of the parameters $\lambda$ and $\mu$, this scheme reproduces polynomials of degree 1. Moreover it reproduces $\mathcal{P}_{2}$ if and only if $\lambda=-\frac{1}{8}$, and $\mathcal{P}_{3}$ if also $\mu=-\frac{1}{2}$.

Applying the de Rham transform, we end up with the dual approximating Hermite subdivision scheme having stationary counterpart $S_{\overline{\mathcal{A}}}$. The non-zero entries of its mask $\overline{\mathcal{A}}$ are given by

$$
\begin{aligned}
& \overline{\mathbf{A}}(-2)=\frac{1}{8}\left[\begin{array}{cc}
2+4 \lambda(1-\mu) & 4 \lambda+2 \lambda \mu \\
4-2 \mu-2 \mu^{2} & \mu^{2}+8 \lambda(1-\mu)
\end{array}\right], \quad \overline{\mathbf{A}}(-1)=\frac{1}{8}\left[\begin{array}{cc}
6-4 \lambda(1-\mu) & 8 \lambda-2 \lambda \mu \\
4-2 \mu-2 \mu^{2} & \mu^{2}-8 \lambda(1-\mu)+2 \mu
\end{array}\right], \\
& \overline{\mathbf{A}}(0)=\frac{1}{8}\left[\begin{array}{cc}
6-4 \lambda(1-\mu) & -8 \lambda+2 \lambda \mu \\
-4+2 \mu+2 \mu^{2} & \mu^{2}-8 \lambda(1-\mu)+2 \mu
\end{array}\right], \quad \overline{\mathbf{A}}(1)=\frac{1}{8}\left[\begin{array}{cc}
2+4 \lambda(1-\mu) & -4 \lambda-2 \lambda \mu \\
-4+2 \mu+2 \mu^{2} & \mu^{2}+8 \lambda(1-\mu)
\end{array}\right] .
\end{aligned}
$$

Since $H_{\bar{A}}$ satisfies the spectral condition of order 2 , we can construct the mask $\overline{\mathcal{B}}=\{\overline{\mathbf{B}}(\alpha), \alpha \in \mathbb{Z}\}$ supported on $[-1,1]$ such that

$$
\left[\begin{array}{cc}
z^{-1}-1 & -1 \\
0 & 1
\end{array}\right] \overline{\mathcal{A}}^{*}(z)=\frac{1}{2} \overline{\mathcal{B}}^{*}(z)\left[\begin{array}{cc}
z^{-2}-1 & -1 \\
0 & 1
\end{array}\right] .
$$


Moreover, due to the fact that the common eigenvector of $\overline{\mathbf{B}}_{e}:=\sum_{\alpha \in \mathbb{Z}} \overline{\mathbf{B}}(2 \alpha)$ and $\overline{\mathbf{B}}_{o}:=\sum_{\alpha \in \mathbb{Z}} \overline{\mathbf{B}}(2 \alpha+$ 1 ) is $[0,1]^{T}$, in view of the results in [1], the difference mask of $\overline{\mathcal{B}}$, hereinafter denoted by $\widetilde{\mathcal{B}}$, can be computed as

$$
\left[\begin{array}{cc}
1 & 0 \\
0 & z^{-1}-1
\end{array}\right] \overline{\mathcal{B}}^{*}(z)=\stackrel{\sim}{\mathcal{B}}^{*}(z)\left[\begin{array}{cc}
1 & 0 \\
0 & z^{-2}-1
\end{array}\right]
$$

The non-zero entries of the mask $\stackrel{\widetilde{\mathcal{B}}}{\text { are }}$

$$
\begin{aligned}
& \widetilde{\mathbf{B}}(-1)=\frac{1}{4}\left[\begin{array}{cc}
2-4 \lambda(\mu-1) & 2 \lambda(\mu+2) \\
-2 \mu^{2}-2 \mu+4 & \mu^{2}-8 \lambda(\mu-1)
\end{array}\right], \\
& \widetilde{\mathbf{B}}(0)=\frac{1}{4}\left[\begin{array}{cc}
2 \mu^{2}+2 \mu+8 \lambda(\mu-1) & 4 \lambda(\mu-1)-\mu^{2} \\
0 & 2 \mu+16 \lambda(\mu-1)
\end{array}\right], \\
& \widetilde{\overline{\mathbf{B}}}(1)=\frac{1}{4}\left[\begin{array}{cc}
2 \mu^{2}+2 \mu-4 \lambda(\mu-1)-2 & 2-2 \mu-6 \lambda \mu-\mu^{2} \\
2 \mu^{2}+2 \mu-4 & 4-2 \mu-8 \lambda(\mu-1)-\mu^{2}
\end{array}\right] .
\end{aligned}
$$

To show that the Hermite subdivision scheme $H_{\overline{\bar{\lambda}}}$ is $H C^{2}$ (namely, accordingly to Definition $1, S_{\overline{\bar{\gamma}}}$ generates a $C^{2}$ limit function) we show that $S_{\bar{B}}$ is $C^{1}$. Following Theorem 3 this is done by proving that its divided difference scheme $S_{\widetilde{C}}$, with $\widetilde{\mathcal{C}}=2 \widetilde{\overline{\mathcal{B}}}$, is $C^{0}$. In view of the results in [1], a necessary condition for $S_{\widetilde{C}}$ to be convergent is that $\widetilde{\mathbf{C}}_{e}$ and $\widetilde{\mathbf{C}}_{o}$ have at least a common eigenvector associated with the eigenvalue 1 . Since,

$$
\widetilde{\mathbf{C}}_{o}=\frac{1}{2}\left[\begin{array}{cc}
8 \lambda(1-\mu)+2 \mu+2 \mu^{2} & 4 \lambda(1-\mu)-\mu^{2}-2 \mu+2 \\
0 & 4-2 \mu+16 \lambda(1-\mu)
\end{array}\right]
$$

and

$$
\widetilde{\mathbf{C}}_{e}=\frac{1}{2}\left[\begin{array}{cc}
-8 \lambda(1-\mu)+2 \mu+2 \mu^{2} & -4 \lambda(1-\mu)-\mu^{2} \\
0 & 2 \mu-16 \lambda(1-\mu)
\end{array}\right]
$$

they have a common eigenvalue 1 if and only if the following conditions are fulfilled:

(i) $4 \lambda(1-\mu)+\mu+\mu^{2}=1$ or $2-\mu+8 \lambda(1-\mu)=1$, for $\widetilde{\mathbf{C}}_{o}$

and

$$
\text { (ii) } \quad-4 \lambda(1-\mu)+\mu+\mu^{2}=1 \text { or } \mu-8 \lambda(1-\mu)=1, \text { for } \widetilde{\mathbf{C}}_{e} .
$$

To make the second equation in $(i)$ and $(i i)$ satisfied, we set $\lambda=-\frac{1}{8}$. Under this assumption the joint 1-eigenspace $\mathcal{E}_{\widetilde{c}}$ is the eigenvector $[1,2]^{T}$.

In order to apply the theory in [1] we continue by applying the similarity transform $\mathbf{S}=\left[\begin{array}{ll}1 & 0 \\ 2 & 1\end{array}\right]$ to the matrix mask $\widetilde{C}$ ending up with the matrix mask $\mathcal{C}=\left\{\mathbf{C}(\alpha)=\mathbf{S}^{-1} \widetilde{\mathbf{C}}(\alpha) \mathbf{S}, \alpha \in \mathbb{Z}\right\}$ such that $\mathcal{E}_{C}=[1,0]^{T}$. The non-zero elements of the mask $\mathcal{C}$ are

$$
\mathbf{C}(-1)=\frac{1}{4}\left[\begin{array}{cc}
1 & -1-\frac{1}{2} \mu \\
2 & 3 \mu+2 \mu^{2}
\end{array}\right], \quad \mathbf{C}(0)=\frac{1}{4}\left[\begin{array}{cc}
4 & 1-\mu-2 \mu^{2} \\
0 & 2+2 \mu+4 \mu^{2}
\end{array}\right], \quad \mathbf{C}(1)=\frac{1}{4}\left[\begin{array}{cc}
3 & 4-\frac{5}{2} \mu-2 \mu^{2} \\
-2 & -2+3 \mu+2 \mu^{2}
\end{array}\right] .
$$

For $\mathcal{C}$ we construct the difference mask $\mathcal{E}=\{\mathbf{E}(\alpha), \alpha \in \mathbb{Z}\}$ satisfying

$$
\left[\begin{array}{cr}
z^{-1}-1 & 0 \\
0 & 1
\end{array}\right] \mathcal{C}^{*}(z)=\mathcal{E}^{*}(z)\left[\begin{array}{cc}
z^{-2}-1 & 0 \\
0 & 1
\end{array}\right]
$$

whose non-zero entries are 


$$
\begin{aligned}
& \mathbf{E}(-1)=\frac{1}{8}\left[\begin{array}{cc}
0 & -\mu-2 \\
0 & 0
\end{array}\right], \quad \mathbf{E}(0)=\frac{1}{8}\left[\begin{array}{cc}
0 & -4 \mu^{2}-\mu+4 \\
0 & 4 \mu^{2}+6 \mu
\end{array}\right], \\
& \mathbf{E}(1)=\frac{1}{8}\left[\begin{array}{cc}
2 & 6-3 \mu \\
0 & 8 \mu^{2}+4 \mu+4
\end{array}\right], \quad \mathbf{E}(2)=\frac{1}{8}\left[\begin{array}{ll}
6 & 4 \mu^{2}+5 \mu-8 \\
4 & 4 \mu^{2}+6 \mu-4
\end{array}\right] .
\end{aligned}
$$

Now we look for a range of $\mu$ such that for a small $\bar{k}$ (say $\bar{k}<10$ ) the iterated subdivision scheme $S_{x}^{\bar{k}}$ is contractive, that is $\left\|S_{\varepsilon}^{\bar{k}}\right\|_{\infty}<1$. For $\mu \in[-0.7,0.425]$ we find $\left\|S_{\varepsilon}^{4}\right\|_{\infty}<1$, so that we can conclude that the scheme $H_{\bar{\Omega}}$ is $H C^{2}$, while for $\mu$ in the same range $H_{\Omega}$ is only $H C^{1}$.

Figures 1 and 2 are to compare the basic matrix limit functions of $H_{A}$ and $H_{\bar{A}}$ for the parameter choice $\mu=-\frac{1}{2}$.
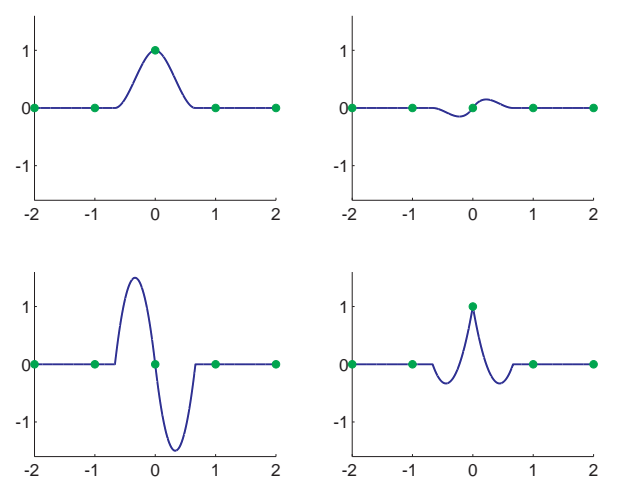

Figure 1: Basic matrix limit function of the interpolatory Hermite subdivision scheme $H_{\Re}$ with $\mu=-\frac{1}{2}$.
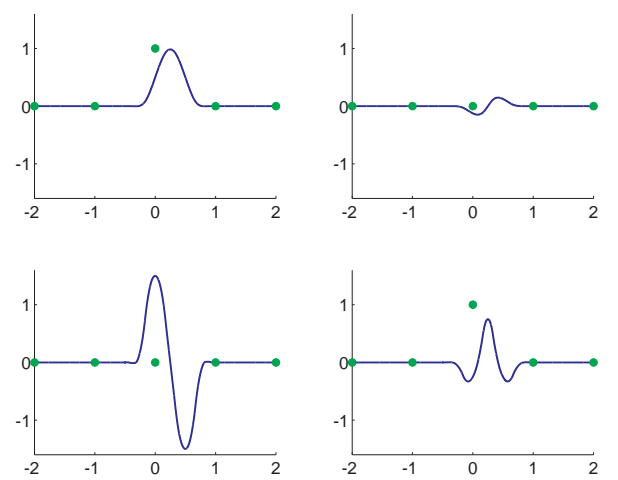

Figure 2: Basic matrix limit function of the non-interpolatory dual Hermite subdivision scheme $H_{\bar{\Omega}}$ with $\mu=-\frac{1}{2}$.

\subsection{A dual non-interpolatory $H C^{3}$ Hermite subdivision scheme of order 3}

In this case we start with the interpolatory Hermite subdivision scheme of order $d+1=3$ proposed by Merrien in [16]. This is a 2-point subdivision scheme depending on several real parameters. The 
non-zero matrices of the mask $\mathcal{A}$ of its stationary counterpart are

$\mathbf{A}(-1)=\mathbf{D}\left[\begin{array}{lll}\alpha_{1} & \alpha_{2} & \alpha_{3} \\ \beta_{1} & \beta_{2} & \beta_{3} \\ \gamma_{1} & \gamma_{2} & \gamma_{3}\end{array}\right], \quad \mathbf{A}(0)=\mathbf{D}, \quad \mathbf{A}(1)=\mathbf{D}\left[\begin{array}{ccc}\alpha_{1} & -\alpha_{2} & \alpha_{3} \\ -\beta_{1} & \beta_{2} & -\beta_{3} \\ \gamma_{1} & -\gamma_{2} & \gamma_{3}\end{array}\right]$ where $\mathbf{D}=\left[\begin{array}{ccc}1 & 0 & 0 \\ 0 & \frac{1}{2} & 0 \\ 0 & 0 & \frac{1}{4}\end{array}\right]$.

To guarantee reproduction of degree 3 polynomials, among the free parameters, the following constraints are assumed (see [12]):

$$
\alpha_{1}=\frac{1}{2}, \quad \gamma_{1}=0, \quad \beta_{2}=\frac{1-\beta_{1}}{2}, \quad \gamma_{3}=\frac{1-\gamma_{2}}{2}, \quad \alpha_{3}=\frac{-1-8 \alpha_{2}}{16}, \beta_{3}=\frac{2 \beta_{1}-3}{24} .
$$

Since the mask under consideration is now depending only on $\alpha_{2}, \beta_{1}, \gamma_{2}$, for shortness we rename the free parameters as $\alpha, \beta, \gamma$, respectively, and denote by $\mathcal{R}$ their range of variation given in [12] to ensure $H C^{2}$-continuity of the subdivision scheme. Therefore the mask we consider reads as

$\mathbf{A}(-1)=\left[\begin{array}{ccc}\frac{1}{2} & \alpha & \frac{-1-8 \alpha}{16} \\ \frac{\beta}{2} & \frac{1-\beta}{4} & \frac{2 \beta-3}{48} \\ 0 & \frac{\gamma}{4} & \frac{1-\gamma}{8}\end{array}\right], \quad \mathbf{A}(0)=\left[\begin{array}{ccc}1 & 0 & 0 \\ 0 & \frac{1}{2} & 0 \\ 0 & 0 & \frac{1}{4}\end{array}\right], \quad \mathbf{A}(1)=\left[\begin{array}{ccc}\frac{1}{2} & -\alpha & \frac{-1-8 \alpha}{16} \\ -\frac{\beta}{2} & \frac{1-\beta}{4} & -\frac{2 \beta-3}{48} \\ 0 & -\frac{\gamma}{4} & \frac{1-\gamma}{8}\end{array}\right],(\alpha, \beta, \gamma) \in \mathcal{R}$.

Applying the de Rham transform we end up with the dual approximating Hermite subdivision scheme $H_{\overline{\mathbb{A}}}$. The non-zero entries of the mask $\overline{\mathcal{A}}$ are $\overline{\mathbf{A}}(-2), \overline{\mathbf{A}}(-1), \overline{\mathbf{A}}(0), \overline{\mathbf{A}}(1)$ and the associated symbol $\overline{\mathcal{A}}^{*}(z)$ is a $3 \times 3$ matrix with polynomial entries $\bar{a}_{i j}^{*}(z), i, j=0,1,2$, given by

$$
\begin{aligned}
\bar{a}_{00}^{*}(z) & =\frac{1}{384}\left((192 \alpha \beta+96) z^{-2}+(288-192 \alpha \beta) z^{-1}+(288-192 \alpha \beta)+(192 \alpha \beta+96) z\right), \\
\bar{a}_{01}^{*}(z) & =\frac{1}{384}\left((288 \alpha-96 \alpha \beta-6 \gamma(8 \alpha+1)) z^{-2}+(288 \alpha+96 \alpha \beta-6 \gamma(8 \alpha+1)) z^{-1}\right. \\
& +(6 \gamma(8 \alpha+1)-96 \alpha \beta-288 \alpha)+(96 \alpha \beta-288 \alpha+6 \gamma(8 \alpha+1)) z), \\
\bar{a}_{02}^{*}(z) & =\frac{1}{384}\left((3 \gamma-144 \alpha+16 \alpha \beta+24 \alpha \gamma-15) z^{-2}+(3 \gamma-144 \alpha-16 \alpha \beta+24 \alpha \gamma-21) z^{-1}\right. \\
& +(3 \gamma-144 \alpha-16 \alpha \beta+24 \alpha \gamma-21)+(3 \gamma-144 \alpha+16 \alpha \beta+24 \alpha \gamma-15) z), \\
\bar{a}_{10}^{*}(z) & =\frac{1}{384}\left((-96 \beta(\beta-3)) z^{-2}+(-96 \beta(\beta-3)) z^{-1}+(96 \beta(\beta-3))+(96 \beta(\beta-3)) z\right), \\
\bar{a}_{11}^{*}(z) & =\frac{1}{384}\left(\left(48(\beta-1)^{2}+384 \alpha \beta+4 \gamma(2 \beta-3)\right) z^{-2}+\left(48 \beta^{2}-384 \alpha \beta-192 \beta-4 \gamma(2 \beta-3)+144\right) z^{-1}\right. \\
& \left.+\left(48 \beta^{2}-384 \alpha \beta-192 \beta-4 \gamma(2 \beta-3)+144\right)+\left(48(\beta-1)^{2}+384 \alpha \beta+4 \gamma(2 \beta-3)\right) z\right), \\
\bar{a}_{12}^{*}(z) & =\frac{1}{384}\left(\left(6 \gamma-192 \alpha \beta-4 \beta \gamma-8 \beta^{2}-18\right) z^{-2}+\left(48 \beta-6 \gamma+192 \alpha \beta+4 \beta \gamma-8 \beta^{2}-18\right) z^{-1}\right. \\
& \left.+\left(6 \gamma-48 \beta-192 \alpha \beta-4 \beta \gamma+8 \beta^{2}+18\right)+\left(192 \alpha \beta-6 \gamma+4 \beta \gamma+8 \beta^{2}+18\right) z\right), \\
\bar{a}_{20}^{*}(z) & =\frac{1}{384}\left((192 \beta \gamma) z^{-2}+(-192 \beta \gamma) z^{-1}+(-192 \beta \gamma)+(192 \beta \gamma) z\right), \\
\bar{a}_{21}^{*}(z) & =\frac{1}{384}\left((-48 \gamma(2 \beta+\gamma-3)) z^{-2}+(48 \gamma(2 \beta-\gamma+3)) z^{-1}\right. \\
& +(-48 \gamma(2 \beta-\gamma+3))+(48 \gamma(2 \beta+\gamma-3)) z), \\
\bar{a}_{22}^{*}(z)= & \frac{1}{384}\left(\left(24 \gamma^{2}+8 \gamma(2 \beta-9)+24\right) z^{-2}+\left(24 \gamma^{2}-8 \gamma(2 \beta+9)+72\right) z^{-1}\right. \\
+ & \left.\left(24 \gamma^{2}-8 \gamma(2 \beta+9)+72\right)+\left(24 \gamma^{2}+8 \gamma(2 \beta-9)+24\right) z\right),
\end{aligned}
$$


where the coefficients of $\bar{a}_{i j}^{*}(z)$ are the $(i, j)$-entries of the matrices $\overline{\mathbf{A}}(-2), \overline{\mathbf{A}}(-1), \overline{\mathbf{A}}(0), \overline{\mathbf{A}}(1)$, respectively. Since $H_{\bar{\lambda}}$ satisfies the spectral condition of order 3 we can construct the mask $\overline{\mathcal{B}}$ supported on $[-1,1]$ such that

$$
\left[\begin{array}{ccc}
z^{-1}-1 & -1 & -\frac{1}{2} \\
0 & z^{-1}-1 & -1 \\
0 & 0 & 1
\end{array}\right] \overline{\mathcal{A}}^{*}(z)=\frac{1}{4} \overline{\mathcal{B}}^{*}(z)\left[\begin{array}{ccc}
z^{-2}-1 & -1 & -\frac{1}{2} \\
0 & z^{-2}-1 & -1 \\
0 & 0 & 1
\end{array}\right] .
$$

Like in the previous example, we compute from $\overline{\mathcal{B}}$ the difference mask $\widetilde{\bar{B}}$ whose non-zero entries are $\widetilde{\overline{\mathbf{B}}}(-1), \widetilde{\mathbf{B}}(0), \widetilde{\overline{\mathbf{B}}}(1)$, and the associated symbol $\widetilde{\overline{\mathcal{B}}}^{*}(z)$ is given by

$$
\begin{aligned}
& \widetilde{\bar{b}}_{00}^{*}(z)=\frac{1}{96}\left((192 \alpha \beta+96) z^{-1}+\left(96 \beta^{2}-384 \alpha \beta-96 \beta \gamma-288 \beta+192\right)\right. \\
&\left.+\left(192 \alpha \beta-288 \beta+96 \beta \gamma+96 \beta^{2}+96\right) z\right), \\
& \widetilde{\bar{b}}_{01}^{*}(z)=\frac{1}{96}\left((288 \alpha-96 \alpha \beta-2 \gamma(24 \alpha+3)) z^{-1}\right. \\
&+\left(96 \beta-192 \alpha \beta-48 \beta^{2}+24 \gamma^{2}+2 \gamma(20 \beta-30)-48\right) \\
&\left.+\left(192 \beta-288 \alpha+288 \alpha \beta-2 \gamma(20 \beta-24 \alpha+39)-48 \beta^{2}+24 \gamma^{2}-48\right) z\right), \\
& \widetilde{\bar{b}}_{02}^{*}(z)=\frac{1}{96}\left(((24 \alpha+3) \gamma+16 \alpha \beta-144 \alpha-15) z^{-1}+\left((30-4 \beta) \gamma-12 \gamma^{2}+8 \beta^{2}+160 \alpha \beta\right)\right. \\
&\left.+\left((4 \beta-24 \alpha+39) \gamma-12 \gamma^{2}+144 \alpha-48 \beta-176 \alpha \beta+8 \beta^{2}+15\right) z\right), \\
& \widetilde{\bar{b}}_{10}^{*}(z)=\frac{1}{96}\left((-96 \beta(\beta-3)) z^{-1}+(-192 \beta \gamma)+(96 \beta(\beta+2 \gamma-3)) z\right), \\
& \widetilde{\bar{b}}_{11}^{*}(z)=\frac{1}{96}\left(\left((8 \beta-12) \gamma+384 \alpha \beta-96 \beta+48 \beta^{2}+48\right) z^{-1}\right. \\
&+\left(48 \gamma^{2}+(80 \beta-120) \gamma-96 \beta-768 \alpha \beta+96\right) \\
&\left.+\left(48 \gamma^{2}-(88 \beta+156) \gamma+192 \beta+384 \alpha \beta-48 \beta^{2}+48\right) z\right), \\
& \widetilde{\bar{b}}_{12}^{*}(z)=\frac{1}{96}\left(\left((6-4 \beta) \gamma-8 \beta^{2}-192 \alpha \beta-18\right) z^{-1}+\left(-24 \gamma^{2}+(60-8 \beta) \gamma+48 \beta+384 \alpha \beta-24\right)\right. \\
&\left.+\left((12 \beta+78) \gamma-24 \gamma^{2}+8 \beta^{2}-192 \alpha \beta-48 \beta-6\right) z\right), \\
& \widetilde{\bar{b}}_{20}^{*}(z)=\frac{1}{96}\left((192 \beta \gamma) z^{-1}+(-384 \beta \gamma)+(192 \beta \gamma) z\right), \\
& \widetilde{\bar{b}}_{21}^{*}(z)=\frac{1}{96}\left((48 \gamma(-2 \beta-\gamma+3)) z^{-1}+(192 \beta \gamma)+(48 \gamma(-2 \beta+\gamma-3)) z\right), \\
& \widetilde{\bar{b}}_{22}^{*}(z)=\frac{1}{96}\left(\left(24 \gamma^{2}+(16 \beta-72) \gamma+24\right) z^{-1}+(48-32 \beta \gamma)+\left(-24 \gamma^{2}+(16 \beta+72) \gamma+24\right) z\right) .
\end{aligned}
$$

To see weather the Hermite subdivision scheme $H_{\bar{\Omega}}$ is $H C^{3}$ we use Theorem 3 and check if $S_{\bar{B}}$ is $C^{1}$, which is equivalent to the fact that its divided difference scheme $S_{\widetilde{\mathcal{C}}}$, with $\widetilde{\mathcal{C}}=2 \widetilde{\overline{\mathcal{B}}}$, is $C^{0}$. Since the joint 1-eigenspace of $\widetilde{\mathbf{C}}_{e}$ and $\widetilde{\mathbf{C}}_{o}$ is $\mathcal{E}_{\tilde{\mathcal{C}}}=[1,3,6]^{T}$, we apply the similarity transform $\mathbf{C}(\alpha)=\mathbf{S}^{-1} \widetilde{\mathbf{C}}(\alpha) \mathbf{S}$ for $\alpha=-1,0,1$ with

$$
\mathbf{S}=\left(\begin{array}{lll}
1 & 0 & 0 \\
3 & 1 & 0 \\
6 & 0 & 1
\end{array}\right)
$$


so that $\mathcal{E}_{c}=[1,0,0]^{T}$. To show that $S_{c}$ is $C^{0}$, we construct the difference mask $\mathcal{E}$ via

$$
\left[\begin{array}{ccc}
z^{-1}-1 & 0 & 0 \\
0 & 1 & 0 \\
0 & 0 & 1
\end{array}\right] \mathcal{C}^{*}(z)=\mathcal{E}^{*}(z)\left[\begin{array}{ccc}
z^{-2}-1 & 0 & 0 \\
0 & 1 & 0 \\
0 & 0 & 1
\end{array}\right]
$$

which yields

$$
\begin{aligned}
& e_{00}^{*}(z)=\frac{1}{48}\left(6 z+42 z^{2}\right), \\
& e_{01}^{*}(z)=\frac{1}{48}\left((288 \alpha-6(8 \alpha+1) \gamma-96 \alpha \beta) z^{-1}\right. \\
& +\left(24 \gamma^{2}+(48 \alpha+40 \beta-54) \gamma+96 \beta-288 \alpha-96 \alpha \beta-48 \beta^{2}-48\right) \\
& +((48 \alpha-80 \beta-18) \gamma+96 \beta-288 \alpha+480 \alpha \beta) z \\
& \left.+\left((40 \beta-48 \alpha+78) \gamma-24 \gamma^{2}+288 \alpha-192 \beta-288 \alpha \beta+48 \beta^{2}+48\right) z^{2}\right) \text {, } \\
& e_{02}^{*}(z)=\frac{1}{48}\left(((24 \alpha+3) \gamma+16 \alpha \beta-144 \alpha-15) z^{-1}\right. \\
& +\left((27-4 \beta-24 \alpha) \gamma-12 \gamma^{2}+8 \beta^{2}+144 \alpha \beta+144 \alpha+15\right) \\
& +((8 \beta-24 \alpha+9) \gamma+144 \alpha-48 \beta-336 \alpha \beta+15) z \\
& \left.+\left(12 \gamma^{2}+(24 \alpha-4 \beta-39) \gamma+48 \beta-144 \alpha+176 \alpha \beta-8 \beta^{2}-15\right) z^{2}\right), \\
& e_{10}^{*}(z)=\frac{1}{48}\left(18 z^{2}\right) \\
& e_{11}^{*}(z)=\frac{1}{48}\left(\left((144 \alpha+8 \beta+6) \gamma+672 \alpha \beta-96 \beta-864 \alpha+48 \beta^{2}+48\right)\right. \\
& +\left((60-40 \beta) \gamma-24 \gamma^{2}+144 \beta^{2}-192 \alpha \beta-384 \beta+240\right) z \\
& \left.+\left((32 \beta-144 \alpha+78) \gamma-24 \gamma^{2}+864 \alpha-384 \beta-480 \alpha \beta+96 \beta^{2}+192\right) z^{2}\right) \text {, } \\
& e_{12}^{*}(z)=\frac{1}{48}\left(\left(432 \alpha-(72 \alpha+4 \beta+3) \gamma-8 \beta^{2}-240 \alpha \beta+27\right)\right. \\
& +\left(12 \gamma^{2}+(4 \beta-30) \gamma+48 \beta-96 \alpha \beta-24 \beta^{2}-24\right) z \\
& \left.+\left(12 \gamma^{2}+(72 \alpha-39) \gamma+96 \beta-432 \alpha+336 \alpha \beta-16 \beta^{2}-51\right) z^{2}\right), \\
& e_{20}^{*}(z)=\frac{1}{48}\left(108 z^{2}\right) \text {, } \\
& e_{21}^{*}(z)=\frac{1}{48}\left(\left(-48 \gamma^{2}+(288 \alpha-96 \beta+180) \gamma-1728 \alpha+576 \alpha \beta\right)\right. \\
& +\left((360-48 \beta) \gamma-144 \gamma^{2}+1152 \alpha \beta-576 \beta+288 \beta^{2}+288\right) z \\
& \left.+\left((144 \beta-288 \alpha+324) \gamma-96 \gamma^{2}+1728 \alpha-1152 \beta-1728 \alpha \beta+288 \beta^{2}+288\right) z^{2}\right) \text {, } \\
& e_{22}^{*}(z)=\frac{1}{48}\left(\left(24 \gamma^{2}+(16 \beta-144 \alpha-90) \gamma+864 \alpha-96 \alpha \beta+114\right)\right. \\
& +\left(72 \gamma^{2}+(-8 \beta-180) \gamma-48 \beta^{2}-960 \alpha \beta+48\right) z \\
& \left.+\left(48 \gamma^{2}+(144 \alpha-8 \beta-162) \gamma+288 \beta-864 \alpha+1056 \alpha \beta-48 \beta^{2}-66\right) z^{2}\right) \text {. }
\end{aligned}
$$

When $\alpha=-\frac{5}{32}, \gamma=\frac{3}{2}, \beta=2$, we can show that $\exists \bar{n}<10:\left\|S_{x}^{\bar{n}}\right\|_{\infty}<1, \quad$ i.e. $S_{\varepsilon}$ is contractive, namely $S_{c}$ is $C^{0}$ and $H_{\bar{\lambda}}$ is $H C^{3}$. Differently, for the same choice of the free parameters the associated interpolatory Hermite subdivision scheme $H_{\mathfrak{A}}$ is only $H C^{2}$ (see Figures 3 and 4 for a comparison of the basic matrix limit functions of the two schemes). 

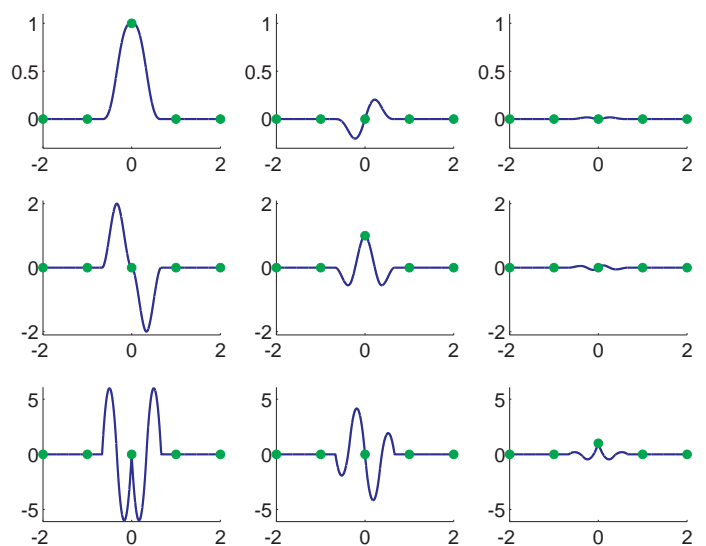

Figure 3: Basic matrix limit function of the interpolatory Hermite subdivision scheme $H_{\imath}$ with $\alpha=-\frac{5}{32}, \gamma=\frac{3}{2}, \beta=2$.
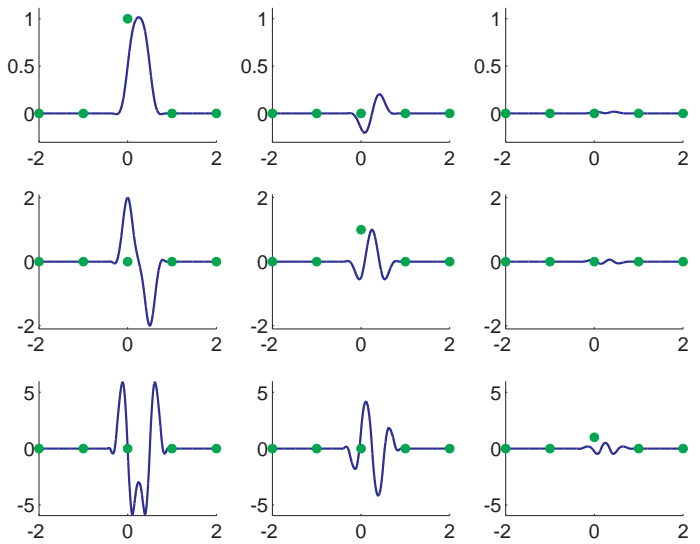

Figure 4: Basic matrix limit function of the non-interpolatory dual Hermite subdivision scheme $H_{\bar{\lambda}}$ with $\alpha=-\frac{5}{32}, \gamma=\frac{3}{2}, \beta=2$.

\section{Conclusions}

In this paper we have presented a novel constructive approach for deriving new dual approximating Hermite subdivision schemes from known Hermite interpolatory schemes. The strategy, called de Rham transform, at each recursion step consists in the double application of an interpolatory Hermite subdivision operator, followed by a decimation step selecting the odd entries only of the obtained vector sequence. Applying the proposed construction to Merrien's interpolatory Hermite schemes in $[16,17]$, two new dual de Rham-type Hermite subdivision schemes are obtained. Their smoothness is analyzed with the help of new theoretical results showing that, with respect to the starting schemes, the regularity is increased by one as well as the support width of the associated basic matrix limit function. 


\section{Acknowledgements}

This work was partially supported by Italian funds from INdAM-GNCS. Special thanks go to the anonymous referees for their useful comments.

\section{References}

[1] M. Charina, C. Conti, T. Sauer, Regularity of multivariate vector subdivision schemes, Numer. Algo. 39 (2005), 97-113.

[2] A. Cohen, N. Dyn, D. Levin, Stability and inter-dependence of matrix subdivision schemes. In: F. Fontanella, K. Jetter, P.-J. Laurent (Eds.), Advanced Topics in Multivariate Approximation, World Scientific Publishing Co., Inc., Singapore, 1993, pp. 33-46.

[3] C. Conti, K. Hormann, Polynomial reproduction for univariate subdivision schemes of any arity, J. Approx. Theory 163 (2011), 413-437.

[4] C. Conti, K. Jetter, A new subdivision method for bivariate splines on the four-directional mesh, J. Comput. Appl. Math. 119 (2000), 936-952.

[5] C. Conti, L. Romani, Algebraic conditions on non-stationary subdivision symbols for exponential polynomial reproduction, J. Comput. Appl. Math. 236 (2011), 543-556.

[6] C. Conti, L. Romani, Dual univariate m-ary subdivision schemes of de Rham-type, J. Math. Anal. Appl. 407 (2013), 443-456.

[7] C. Conti, G. Zimmermann, Interpolatory rank-1 vector subdivision schemes, Comput. Aided Geom. Design 21 (2004), 341-351.

[8] G. de Rham, Sur une courbe plane, J. Math. Pures Appl. 35 (1956), 25-42.

[9] S. Dubuc, J.-L. Merrien, de Rham Transform of a Hermite Subdivision Scheme, Approximation Theory XII, San Antonio 2007, M. Neamtu and L.L. Schumaker (Eds.), 2008, Nashboro Press, Nashville TN, pp. 121-132.

[10] S. Dubuc, J.L. Merrien, Hermite subdivision schemes and Taylor polynomials, Constr. Approx. 29 (2009), 219-245.

[11] N. Dyn, D. Levin, Analysis of Hermite-interpolatory subdivision schemes, In: S. Dubuc and G. Deslauriers (Eds.), Spline Functions and the Theory of Wavelets, Amer. Math. Soc., Providence R. I., 1999, pp. 105-113.

[12] N. Guglielmi, C. Manni, D. Vitale, Convergence analysis of $C^{2}$ Hermite interpolatory subdivision schemes by explicit joint spectral radius formulas, Linear Algebra Appl. 434 (2011), 884-902

[13] B. Han, Vector cascade algorithms and refinable functions in Sobolev spaces, J. Approx. Theory 124 (2003), 44-88.

[14] B. Han, T.P. Yu, B. Piper, Multivariate refinable Hermite interpolant, Math. Comp. 73, no. 248 (2004), 1913-1935.

[15] B. Han, T.P. Yu, Y. Xue, Noninterpolatory Hermite subdivision schemes. Math. Comp. 74, no. 251 (2005), 1345-1367. 
[16] J.-L. Merrien, A family of Hermite interpolants by bisection algorithms, Numer. Algo. 2 (1992), $187-200$.

[17] J.-L. Merrien, Interpolants dHermite $C^{2}$ obtenus par subdivision, M2AN Math. Model. Numer. Anal. 33 (1999), 55-65.

[18] J.-L. Merrien, T. Sauer, A generalized Taylor factorization for Hermite subdivision schemes, J. Comput. Appl. Math. 236, no. 4 (2011), 565-574.

[19] C.A. Micchelli, T. Sauer, On vector subdivision, Math. Z. 229 (1998), 621-674.

[20] L. Romani, A circle-preserving $C^{2}$ Hermite interpolatory subdivision scheme with tension control, Comput. Aided Geom. Design 27 (2010), 36-47. 